\title{
Exploring public engagement with missing person appeals on Twitter
}

\author{
Reka Solymosi, ${ }^{1, *}$ \\ Oana Petcu ${ }^{1}$ \\ Jack Wilkinson ${ }^{2}$
}

${ }^{1}$ School of Social Sciences, University of Manchester, UK

2 Centre for Biostatistics, Manchester Academic Health Science Centre, Division of Population Health, Health Services Research and Primary Care

* Correspondence: Reka Solymosi <reka.solymosi@manchester.ac.uk>

\section{JOURNAL ARTICLE PRE PRINT}

This is the version of this article submitted to a journal for publication. It has not yet been peer reviewed. It may vary from the final published version.

\begin{abstract}
Police agencies globally are seeing an increase in reports of people going missing. These people are often vulnerable, and their safe and early return is a key factor in preventing them from coming to serious harm. One approach to quickly find missing people is to disseminate appeals for information using social media. Yet despite the popularity of twitter-based missing person appeals, presently little is known about how to best construct these messages to ensure they are shared far and wide. This paper aims to build an evidence-base for understanding how police accounts tweet appeals for information about missing persons, and how the public engage with these tweets by sharing them. We analyse 1,008 Tweets made by Greater Manchester Police between the period of 2011 and 2018 in order to investigate what features of the tweet, the twitter account, and the missing person are associated with levels of retweeting. We find that tweets with different choice of image, wording, sentiment, and hashtags vary in how much they are retweeted. Tweets that use custody images have lower retweets than Tweets with regular photos, while tweets asking the question "have you seen...?" and asking explicitly to be retweeted have more engagement in the form of retweets. These results highlight the need for conscientious, evidence-based crafting of missing appeals, and pave the way for further research into the causal mechanisms behind what affects engagement, to develop guidance for police forces worldwide.
\end{abstract}

\section{Introduction}

In the United Kingdom, a missing person is defined as a person of any age whose whereabouts cannot be established. A person is considered missing until they are located, and their well-being or otherwise is confirmed (Policing 2019). Going missing is associated with vulnerabilities such as mental health (Stevenson et al. 2013) and Alzheimers in adults (Petonito et al. 2012), and criminal and sexual exploitation in children (Sharp-Jeffs 2016; Williams and Finlay 2019) particularly those who are often already more vulnerable due to being in care (Biehal, Mitchell, and Wade 2003). The longer people are missing, the greater their exposure to 
negative outcomes such as criminal exploitation, violent victimisation, or suicidal thoughts (Biehal, Mitchell, and Wade 2003; Rees 2011), so finding them as soon as possible can reduce these risks.

The task of finding missing people usually falls to police agencies. In England, missing persons investigations are a bigger cost to police resources than either theft or assault (Shalev Greene and Pakes 2013). In Canada, "compassionate to locate" is the second highest frequency call from citizens to police (Ellingwood 2016). Despite its prevalence, missing persons is not a common area for academic research (compared for example with research on crimes such as domestic violence); a search on BASE (Bielefeld Academic Search Engine - one of the most popular multidisciplinary academic search engines) returns 1,020 hits (out of $154,859,846$ documents) for the terms "missing person" or "missing people, compared to 33,069 hits for the terms "domestic violence" or "domestic abuse".

At least $20 \%$ of those reported missing are not found within 24 hours, and require police intervention to be located, or their welfare (or otherwise) confirmed (Fyfe, Stevenson, and Woolnough 2015). Many police agencies are struggling to cope with the high demands that missing persons investigations create. In the UK, police are no longer required to respond to cases assessed as low-risk (Fyfe, Stevenson, and Woolnough 2015), while in the United States, volunteer programmes have been established to help local police agencies tackle the increasing volume of missing persons cases (Chiefs of Police 2018). As the number of missing people continues to rise each year (National Crime Agency 2019), it is important that police and agencies in search of missing people apply evidence based techniques to make the best use of limited resources in a constrained environment (Crawford 2013; Garside, Silvestri, and Mills 2013).

One approach that is used to locate missing persons is to make appeals for information using social media. A missing person appeal is "communication by those searching for the missing person to a wider network of people who may be able to help locate that person and to the missing person directly" (Holmes 2016, 20). While a media appeal can be regarded by the police as 'being seen to be doing something', it can actually be one of the most important parts of a missing persons enquiry for the police (Fyfe, Stevenson, and Woolnough 2015). If these appeals reach far and wide, they may be seen by either someone with information, or the missing person (Holmes 2016). One study, using a case-control design, even suggests that having tweets posted by police departments can increase the chance of a missing person being found (Tsoi et al. 2018). For the appeals to reach those who may be able to help, they must be widely shared (Lampinen, Peters, and Gier 2012; Tsoi et al. 2018). Yet there is little research on what police can do to promote public engagement and ensure wide sharing. Such an understanding can serve to inform good practice, and maximise the spread of these messages, ultimately contributing to locating missing people, thereby minimising their exposure to associated harms.

In what follows, we consider the available literature on what is known about the public's engagement with social media communications about missing persons. We combine research on Twitter and social media engagement more broadly with research on missing persons appeals in other social and traditional media. We use this review to inform a targeted and purposeful exploration of features we can expect to impact on public engagement with missing persons appeals. We then take a sample of 1,008 tweets about missing persons made by Greater Manchester Police to explore these features and their associations with number of retweets. Our results constitute the first empirical examination of these appeals. We explore the ways in which these appeals are structured, and the implications that factors such as wording, photo choice, and use of social media-specific features such as hashtags may have for public engagement. In our discussion, we draw conclusions about future directions for research to inform good practice in social media appeals for missing persons.

\section{Police strategies on social media}

Police forces across the globe have been making increasing use of social media platforms such as Youtube, Facebook, and Twitter (Crump 2011; Dai et al. 2017). As consumers, they benefit from members of the public sharing information (Earl et al. 2013). As content creators, police benefit from increased accessibility and in cases even improved reputation in local communities (Fernandez, Cano, and Alani 2014). However social media can also be perceived to contain a lot of disinformation and may foster uncertainty (Kwon et 
al. 2016), so it is important that police use social media in a way that fosters engagement and trust. In fact, some research findings suggest that the quality of a police department's media image might have more to do with how they present themselves (including on social media) than with the actual crime rates in their municipality (Lovell 2001).

There is a lot of variation in how the police use social media (Crump 2011). While some guidance exists (eg in the UK the Association for Chief of Police have produced a 'Guidelines on the Safe use of the Internet and Social Media' (ACPO 2013), their implementation is far from uniform across (or even within) forces. In a study comparing Greater Manchester Police (GMP) and London Metropolitan Police (Met) using Twitter during the 2011 riots, Denef, Bayerl, and Kaptein (2013) identified differences such as using an instrumental versus an effective tone, or replying to tips from the public with a 'thanks' (GMP) or not (Met). As a result, people favoured the way in which GMP handled communication, and responded less to the approach taken by the Met. This manifested not only in discussion but also in directly measurable outcomes such as number of followers (Denef, Bayerl, and Kaptein 2013). Crump (2011) identified a UK based typology for police Twitter accounts: 'broadcasters' (who share information), 'local knowledge gatherers' (who share information), and 'community facilitators' (who foster dialogue). Such typologies exist internationally; research in the United States has identified different strategies between forces depending on whether they used it to broadcast, collect intelligence, or for communication and engagement (Meijer and Thaens 2013). Clearly there are many strategies to social media use by police and its important that we understand how these apply to missing appeals and public engagement.

\section{Engagement with Twitter}

In this section we review the literature about what is known to be associated with people's engagement with tweets, to inform our feature selection for this study.

\section{Features of the missing person}

First, in the case of appeals for information about missing persons, it is known that certain factors, ranging from demographic characteristics of the person (race, economic background), newsroom resources, and amount of information that is released by the police all affect media coverage of missing persons (Artwick 2010). While these factors cannot be changed, they are important to understand, so coverage can be increased (where appropriate), and so these can be considered when composing such appeals.

Age of a missing person affects media coverage where younger missing persons are represented in the media more than older ones (Jeanis and Powers 2017). Race of the missing person seems to be indicative of coverage. In the USA, (Rijt et al. 2018) found that non-black children are significantly more likely to receive coverage than non-white children. In Canada, Gilchrist (2010) found missing aboriginal women received 3.5 times less coverage than white women. Gender is also important, whereby missing girls, are less likely to be in the news than missing boys (Min and Feaster 2010). However, intersectionality must be considered, as Artwick (2010) found that appeals of women of certain race receive more civil engagement than men. Featu

\section{Features of the tweet}

Time and timeliness of a tweet may be a factor. Looking at police use of social media in Spain, Fernandez, Cano, and Alani (2014) found that tweets between 8am-4pm have more retweets. Others note that tweets sent on the day of a key event (eg protest, music festival, riot) are associated with more retweets than tweets sent before or after (Stefanone, Egnoto, and Fu 2015; Xu and Zhang 2018). Structurally, the length of post is positively associated with retweets; posts with more words are more likely to get engagement (Fernandez, Cano, and Alani 2014; Xu and Zhang 2018). Punctuation can also make a difference; tweets that end in question marks are more likely to get retweeted than those which end in exclamation marks (Naveed et al. 2011). The use of hashtags shows mixed results. Some studies find hashtags increase retweeting (Suh et al. 
2010; Jenders, Kasneci, and Naumann 2013; Stefanone, Egnoto, and Fu 2015; Van de Velde, Meijer, and Homburg 2015), while others claim the opposite (Lopez 2014; Tsoi et al. 2018). Suh et al. (2010) propose that some hashtags get more retweets than others, so the content of each hashtag as well as the number is important to consider. Other templates or key phrases that seemed to make a difference were including a hyperlink/URL (Zarrella 2009; Suh et al. 2010; Rowe, Angeletous, and Alani 2011; Rowe, Angeletou, and Alani 2011; Van de Velde, Meijer, and Homburg 2015; Xu and Zhang 2018; Cha et al. 2010), expressing gratitude, or asking explicitly to be shared ("please RT") (Lopez 2014).

Sentiment of the tweet also makes a difference for retweetability. Some studies find that messages with positive sentiment increased retweetability (Fernandez, Cano, and Alani 2014; Kim et al. 2016; Stefanone, Egnoto, and Fu 2015) and content with negativity discourages retweeting behaviour (Xu and Zhang 2018), while others found tweets with negative emotions more likely to be shared (Chen and Sakamoto 2013). Xu and Zhang (2018) found that both negative and positive emotion tweets received more retweets than neutral, and Chen and Sakamoto (2013) found that feelings of "alarmed" and "stressed" were also associated with sharing. Ferrara and Yang (2015) found that negative or neutral messages spread faster than positive ones, but positive messages spread broader, and are favourited more. They also found that feelings of "confused", "nothing", "indifferent", "uninterested", or "neutral" were less likely to encourage retweeting (Chen and Sakamoto 2013). We can see that sentiment affects engagement, although it is not obvious what the effects will be.

Studies looking into tone of voice found that content with confident, authentic, informal, and powerful language have higher retweetability (Van de Velde, Meijer, and Homburg 2015; Xu and Zhang 2018). Callsto-action also increase retweeting (Lopez 2014). Specifically for police engagement on social media, Denef, Bayerl, and Kaptein (2013) found using a 'human approach' in communication with public was appreciated. Many studies consider emotional compared to rational tone tweets. By 'rational' we mean that we consider these tweets to be factual or unemotive. We use the term 'rational' as this is consistent with the cited literature. Xu and Zhang (2018) find emotional language has higher retweetability, while Lopez (2014) concludes that emotional content is least likely to be retweeted. In any case tone is important to consider.

Finally the inclusion of useful information has been associated with increased retweets (Lopez 2014), and the inclusion of photos is important both for retweeting (Lopez 2014; Cvetojevic and Hochmair 2018; Xu and Zhang 2018) and for people being able to identify the missing person in the case of such an appeal (Tsoi et al. 2018). It is important not only that there is an image, but positive valence associated with the image (Stefanone, Egnoto, and Fu 2015).

\section{Features of the account}

Besides tweet content, features of the account might influence numbers of retweets. Number of followers has been positively associated with retweets (Suh et al. 2010; Hong, Dan, and Davison 2011; Cha et al. 2010; Jenders, Kasneci, and Naumann 2013; Kim et al. 2016; Stefanone, Egnoto, and Fu 2015; Van de Velde, Meijer, and Homburg 2015; Xu and Zhang 2018), while the age of the account is negatively related to retweets (Suh et al. 2010; Van de Velde, Meijer, and Homburg 2015). The number of tweets from each account shows mixed effects, either showing no significant relationship (Suh et al. 2010), more posts associated with lower odds of being retweeted (Van de Velde, Meijer, and Homburg 2015), or more posts associated with more followers and therefore more retweets(Crump 2011). A key factor in people sharing information on Twitter is whether they believe it to be from a trusted source (Tapia et al. 2011), for example an account of someone in a position of authority such as the police. Because people know the source, they are more likely to trust the validity of the information, and ultimately share tweets (Juncu and Glorney 2019). Since police sharing of appeals has such importance (Tsoi et al. 2018), we consider tweets from police accounts.

Table 1 summarises the features identified by previous literature as important. 
Table 1: Features in the literature

\begin{tabular}{ll}
\hline Element & Feature \\
\hline Features of the missing person & Race/ ethnic appearance, Gender, Age \\
Features of the tweet & Time and timeliness, Post length, Punctuation and hashtags, \\
& $\begin{array}{l}\text { Templates, Sentiment, Tone, Useful information, Photo } \\
\text { (presence and valence) }\end{array}$ \\
Features of the account & $\begin{array}{l}\text { Number of followers, Age of account, Tweeting activity, Trusted } \\
\text { source }\end{array}$ \\
\hline
\end{tabular}

\section{Methods}

\section{Analysis}

To achieve this, we present descriptive analysis, comprising numerical and graphical summaries of 1,008 tweets made by Greater Manchester Police. Additionally, to account for exposure (since the tweets had been "out there" to be retweeted for different lengths of time) we perform an additional multivariable analysis, in which we include all features in a Poisson regression model (Hilbe 2014). This allows us to account for the varying levels of exposure (age) of the tweets by introducing it as an offset term (Yan et al. 2009). Further, as there is evidence of overdispersion in our model, we use a model with scaled standard error (overdispersed Poisson model). We stress that the model is intended to be descriptive, exploratory, and associational only, as our data do not allow us to elucidate the causal relationships between features and outcome. We discuss this more in the next section, and in our discussion.

We focus on Twitter partially because of the relative ease of accessing publicly available data about the sharing of these appeals, and partly because Twitter is a particularly useful platform for widely circulating and sharing information (Kim et al. 2016). While different platforms may behave differently, previous research suggests that there may be not too much of a difference in sharing behaviour between those who use Facebook and those on Twitter (Juncu and Glorney 2019). We chose police Twitter accounts due to the high credibility as discussed above, and narrowed the study to focus on Greater Manchester Police, as they have been identified as effective communicators via Twitter by previous work (see Denef, Bayerl, and Kaptein (2013)). Further, the area they police is particularly affected by missing persons. Greater Manchester have the highest rate for missing adults(3.7 per 1,000 population), and second highest rate for missing children (6 per 1,000 population) (National Crime Agency 2017) in the UK. It was therefore an ideal sample of tweets to consider for this research, from which implications can be adopted to police forces as well as other agencies involved with missing persons appeals nationally and internationally.

\section{Data}

To collect data, 56 Greater Manchester Police Twitter accounts were identified (Police 2019). For each account, their most recent 3200 tweets (the maximum allowed by the free Twitter Application Programme Interface (API)) were queried using the Twitter API. As not all accounts had this many tweets, we collected a database of 169,438 tweets. From this, tweets that contain the words "missing", "last seen" or "searching" were programmatically queried. A preliminary reading of all tweets revealed at least one of these terms to be included in all tweets about missing persons. This resulted in 3,239 tweets, which were further filtered manually, with a coder eliminating duplicates and tweets not about missing persons (missing pet, found person, other topic). This resulted in a final dataset of 1,008 unique appeals for information about missing persons made between 1st September 2011 and 10th January 2019 (date of data collection).

There are a few issues to note about our sample. First, the day of data collection was arbitrarily selected, and provides only a cross sectional picture of retweets into missing persons appeals. Secondly, it is general guidance for police Twitter accounts to remove appeals once the person is found. Since these deleted tweets do not form part of our sample and deletion is associated with a person being found, and since our examined 
features may influence the likelihood that a person is found, our sample is subject to selection bias. The extent and influence of this cannot be known from the sample. However, we found that our sample contained appeals about people who had since been found. We cannot know how many, as we have no reference data set of actual outcomes for the missing persons, so we cannot know how this affects who is and is not present in our sample (found or still missing). Consequently, we do not attempt to draw strong causal conclusions from the data, but offer a descriptive analysis, providing insight into police tweeting and public engagement behaviours, building a foundation of empirical evidence to inform future prospective studies.

\section{Coding}

To operationalise the factors associated with retweeting, we used manual coding and automated feature extraction, driven by our literature review. Our aim was to explore the volume of retweets for these different features by coding them in the tweets. For manual coding, a codebook was developed to guide to coding process (Guest, MacQueen, and Namey 2011). A single independent coder coded the tweets for each of the variables in the code book. In some cases, the coding was a matter of extracting values from the text or categorising images into groups. When coding for sentiment, tone, template, and hashtag type, coding followed a thematic analysis process, whereby tweets which followed similar structures or conveyed similar meanings, were identified and labeled with codes (Graneheim and Lundman 2004) to identify key themes and categories (Smedley and Coulson 2018). Once the primary coder had finished coding, the secondary coder reviewed the codes to assess the connection between the raw text and codes (Guest, MacQueen, and Namey 2011). Then a feedback discussion between the two coders was used to revise definitions and recode where necessary (Guest, MacQueen, and Namey 2011). Below we describe in detail the coding of all our variables.

\section{Features of the missing person}

The missing person's gender appearance was coded from the photo where available. Where no photo was available, but gender was mentioned in the text of tweet, this was extracted. For race, we considered ethnic appearance, coded from the photo where available. Like with gender, race of the missing person was coded from the text where no photo was available. Due to the difficulty of inferring age from often low quality photos, we did not consider age in this analysis.

\section{Features of the tweet}

\section{Time - Age of tweet, time of tweet}

To code for time of tweet we extracted the hour of day when the tweet was created through the Twitter API. For timeliness we consider the age of the tweet, calculated by subtracting the day when the tweet was created (available from the Twitter API) from the day of data collection.

\section{Length of post}

An automatic function to count the number of characters in the text of each tweet was used to operationalise post length.

\section{Punctuation}

We considered the use of question mark (?), exclamation mark (!), and asterisk $\left(^{*}\right)$ by using an automatic function to count the number of times each one occurred in the tweet. 


\section{Hashtags}

Like punctuation, hashtags were counted by an automatic function to determine whether any were present in the tweet. For type of hashtag, qualitative coding was carried out to group hashtags into different themes, which emerged from the data (eg whether the hashtag referred to a location, an event, etc).

\section{Templates}

To operationalise whether the tweets were authentic/personal or whether they followed some template, thematic analysis was used. Repeat patterns of phrasing were noted by the primary coder. Initially 130 templates were coded, which were subsumed into 11 themes. For example, the templates: "police are concerned", "we are concerned" and "police are growing increasingly concerned", were all deemed to be following the theme "... are concerned...").

\section{Sentiment}

Sentiment was coded both manually and automatically. Manually, the qualitative coder read through messages and categorised them in terms of the feeling that they elicited to the reader. For example, a sentiment that emerged was 'hopeful', the feeling being that of hope that the missing person will be found soon. A message could elicit more than one sentiment. Once all tweets were assigned a sentiment, the secondary coder considered a sample of the coded tweets. Discrepancies were discussed, and an overview of all the sentiments was carried out, removing from consideration sentiments that appeared infrequently. For example, only 3 tweets were coded with the sentiment "uncertain" and only 1 with the sentiment "angry", so these sentiments were not considered in the analysis.

For automatic sentiment coding, we made use of the AFINN sentiment lexicon, as we wanted to develop an average score for each whole tweet, rather than extract sentiment of individual words. To illustrate, we show two tweets in our sample (any word with sentiment has the score in brackets):

Low score of -10 :

"HIGH risk (-2) missing (-2) person appeal;FIRSTNAME LASTNAME, age 78. Last seen leaving his address in Stretford at 14:30 on 07/06/18. Can his photograph be shared $(+1)$ \& people keep any eye out for him, FIRSTNAME suffers(-2) from dementia \& is very vulnerable(-2), lost(-3) confused(-2). Thanks(+2) Pc M."

High score of 4:

"FIRSTNAME LASTNAME from LOCATION is still missing(-2) - can you help $(+2)$ us to locate him please $(+1)$ ? If you have any information no(-1) matter $(+1)$ how small please $(+1)$ contact Police and quote log number 944 of 3 October 2018 Thank $(+2)$ you."

\section{Tone of appeal}

Previous research showed that having an emotional or rational tone affects retweetability. To operationalise this construct, the primary coder assigned each tweet into one of two categories: rational or emotional. An example of each is:

Rational:

"Missing:FIRSTNAME LASTNAME, East Bowling, Bradford http://t.co/Qk59Xfwz \#police"

Emotional: 
"FIRSTNAME LASTNAME has been missing for two months. She was last seen in \#Bury and we are desperate to find her. Her family are beside themselves with worry. https://t.co/ RpxbkzZ1Jv"

A sample of these were validated by the secondary coder, and no further changes were made.

\section{Images}

Literature showed that including a photo increases retweetability, but the sentiment of the photo matters. In the case of missing persons appeals, in some cases, the most recent image of someone available is a custody photo ( $16 \%$ of our sample of appeals used a custody image), which we consider to have negative valence. To assess this, each tweet was coded as having a custody photo, a regular photo, or multiple (regular) photos. Tweets with no photo were also coded as such. Photo quality was also coded on a qualitative scale of bad, average, or good/excellent. The latter was originally two separate categories, but as too few tweets had excellent quality photos, these were grouped together. We did not code here about the appearance of the missing person in the photo (ie their appearance as happy, distressed, etc).

\section{Useful information}

While the length can be one proxy measure for how much useful information is included, a binary variable indicating whether the Twitter message included all useful information in the text, or referred readers to a further link was also created by qualitative annotation. Useful information could be: the place where the missing person was last seen, any distinguishing features (eg: "flower tattoo on right shoulder"). An example of a tweet with no useful information are:

"Have you seen missing FIRST NAME LAST NAME from \#LOCATION?".

While such tweets are usually followed by links, here we considered the importance of including useful information in the body of the tweet. Tweets coded as not containing useful information referred the reader to a link, article, or other tweet for this information. Example phrases from such tweets are:

"See next tweet for her description"

"MEN report: LINK"

\section{Features of the account}

The number of followers for each account at the time of data collection was available through the free Twitter API. To calculate the age of account, the variable 'account create day' (available from free API) was subtracted from the date of data collection. Finally, to calculate tweeting activity, the average number of daily tweets was calculated using the initial database of all tweets (not only missing persons) for each account.

All data wrangling, analysis, and visualisation was done in $\mathrm{R}$ (version 3.6.1) and the code for this is made available on the first author's GitHub page (https://github.com/maczokni/misperTweetsCode/).

\section{Results}

The average number of retweets of tweets in our sample is 46 , median $=7$, but with huge variance (variance: 101165 , standard deviation $(\mathrm{SD})=318)$ and right skew $(\min =0, \max =8756)$. In total there were 122 outliers, identified as tweets with retweet counts that are more than 3 standard deviations from the mean. 
The outliers are interesting in themselves, however there are a range of factors that may affect how these are generated, (ie how some tweets "go viral"). Many of these seem to be cases which receive coverage in the traditional media. Unfortunately this is not something we coded, so we cannot conclude about the role of media coverage. It is interesting to note the low median, indicating that tweets typically do not get many retweets. The upper quartile of retweets is 24 (inter quartile range (IQR) $=2-24$ ), further emphasising that most tweets have low retweets. 86 tweets had no retweets (this is not because they were "too new" to have gotten attention, the "youngest" tweet with 0 retweets is 52 days old), and 99 had only one retweet (the youngest of these 30 days old). That there are so many tweets with no sharing at all is interesting to note, and while was not on the original agenda, something to consider in the future. In any case, we recognise these outliers and use medians in most of the analysis that follows.

\section{Features of the missing person}

320 tweets were about women and 505 about men, the rest were about multiple people (0) or gender was not identifiable. Considering ethnic appearance, 74 tweets were about non-white missing persons, compared to 563 about white. For the rest (371) ethnic appearance was unknown.

Overall, white females had the highest average retweets (Table 2). The lowest mean is for non-white females, however the median is higher for this group than that the median for non-white males, due to some tweets about non-white males having high retweet counts, influencing the mean (the highest for non-white males reaching 462 retweets, while for non-white female missing persons the highest number of retweets is 75 ). Tweets about white missing persons had more retweets (mean: 61, median: 11, SD: 415, IQR: 4 - 31) than tweets about non-white missing persons (mean: 25, median: 5, SD: 66, IQR: 2 - 14). Tweets about missing women had more retweets (mean: 81, median: 10, SD: 542, IQR: 3 - 40) than tweets about missing men (mean: 81, median: 10, SD: 542, IQR: 3 - 40).

Table 2: Cross table of gender and ethnic appearance of missing persons coded from tweets showing the mean, median, sd, iqr of retweets for each group, and the number of tweets in each group.

\begin{tabular}{l|l|r|r|r|r|l|r}
\hline Gender & Ethnic appearance & Median & Mean & N & SD & IQR & Maximum \\
\hline female & non-white & 6 & 14 & 32 & 18 & $2-15.75$ & 75 \\
\hline female & white & 14 & 113 & 198 & 685 & $5-47$ & 8756 \\
\hline female & NA & 6 & 35 & 90 & 89 & $2-28$ & 552 \\
\hline male & non-white & 4 & 34 & 41 & 86 & $2-13$ & 462 \\
\hline male & white & 9 & 34 & 358 & 99 & $3-24$ & 1385 \\
\hline male & NA & 4 & 24 & 106 & 75 & $1-16.5$ & 684 \\
\hline NA & non-white & 3 & 3 & 1 & NA & $3-3$ & 3 \\
\hline NA & white & 11 & 12 & 7 & 10 & $6-13$ & 31 \\
\hline NA & NA & 4 & 23 & 175 & 137 & $1-15$ & 1783 \\
\hline
\end{tabular}

\section{Features of the tweet}

In total, 421 (42\%) of the tweets did not have a photo with them. Of the ones that did have a photo, 159 (27\%) $27.1 \%$ were custody photos ( $16 \%$ of all the tweets). Considering photo type shows that tweets with no photo at all have the lowest median retweets, but not the lowest mean. Tweets with custody photo have the lowest mean retweets. However the median is higher with custody photo than no photo. Tweets with a regular photo have higher average and median retweets in our sample than both no photo and custody photo. Multiple photos have higher mean but not a higher median compared with one regular photo (Table $3)$.

Figure 1 shows the relationship between photo type and retweet count, taking into account gender and ethnic appearance. We include with all figures, error bars, which show the interquartile range (IQR). 


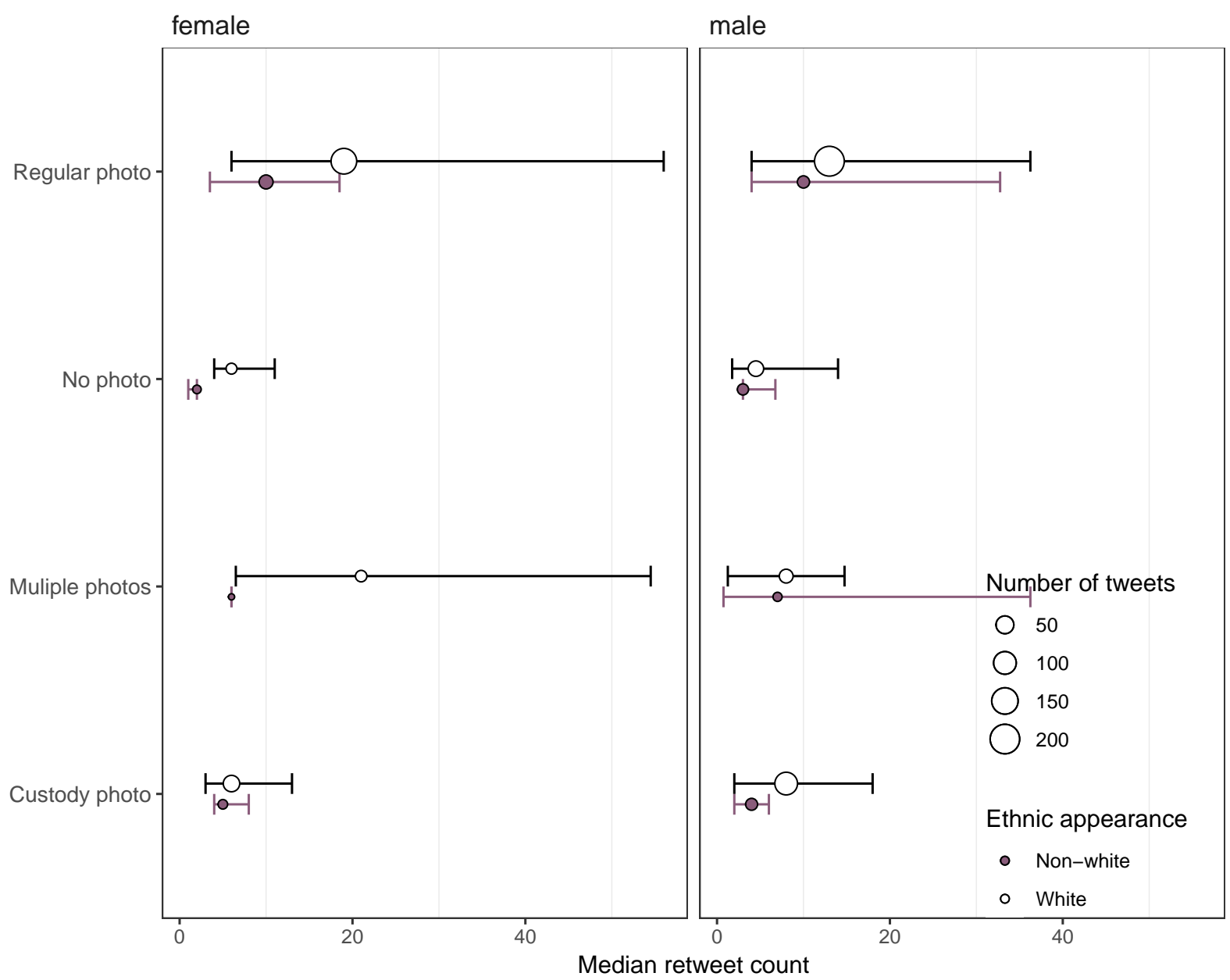

Figure 1: Retweets for different image types by gender and ethnic appearance 
Table 3: Photo type

\begin{tabular}{l|r|r|r|r|l|r}
\hline Photo type & Median & Mean & N & SD & IQR & Maximum \\
\hline Custody photo & 7 & 16 & 159 & 26 & $2.5-16$ & 155 \\
\hline Muliple photos & 12 & 125 & 39 & 638 & $2.5-21.5$ & 3997 \\
\hline No photo & 4 & 24 & 421 & 105 & $1-15$ & 1783 \\
\hline Regular photo & 14 & 74 & 389 & 456 & $5-47$ & 8756 \\
\hline
\end{tabular}

For all groups, tweets with no photo or with a custody image have fewer median retweets than those with a regular (non-custody) photo. Having multiple photos does not seem to get more retweets. In all cases, missing persons who are white have higher median retweets than non-white counterparts.

There is no clear pattern to show that tweets which have better quality photos have more retweets (Table $4)$.

Table 4: Photo quality

\begin{tabular}{l|r|r|r|r|l|r}
\hline Photo quality & Median & Mean & N & SD & IQR & Maximum \\
\hline average & 8 & 97 & 213 & 665 & $3-24$ & 8756 \\
\hline bad & 10 & 35 & 126 & 76 & $3-30$ & 510 \\
\hline good/excellent & 13 & 44 & 242 & 89 & $4-46.75$ & 698 \\
\hline
\end{tabular}

Interestingly though, for men, as photo quality improves, the difference in median retweets for tweets about white and non-white missing persons increases (Figure 2).

There is a weak correlation between days since tweet was created and retweet count (Pearson's productmoment correlation $=0.073$, p-value $=0.021)$. There is no clear difference in median retweets between appeals made in different hours of day (Figure 3) .

Considering the written content of the tweets, length of post does not seem to make a difference to retweets; we cannot say that longer (or shorter) posts have more retweets, either before or after Twitter's change of character limit from 140 characters (Pearson's product-moment correlation $=0.026, \mathrm{p}$-value $=0.464$ ) to 280 characters (Pearson's product-moment correlation $=0, \mathrm{p}$-value $=0.995) .413$ of the tweets included all the useful information in the tweet's text, while the rest pointed to links or attachments for further information.

Tweets with the useful information in-text had higher mean and median retweets $($ mean $=58$, median $=8$, standard deviation $=445, \mathrm{IQR}=3-24$ ) than those that relied on links (mean $=37$, median $=7$, standard deviation $=184, \mathrm{IQR}=2-24)$.

Tweets with question marks $(n=143)$ and tweets with hashtags $(n=175)$ have higher median retweets than tweets without these types of punctuation. Tweets with exclamation marks $(\mathrm{n}=40)$ have the same median to those which do not, and tweets using asterisk $(\mathrm{n}=104)$ have lower median retweet than tweets that do not (Figure 4).

In our sample, most tweets have no hashtags at all. Taken together, tweets with at least one hashtag $(\mathrm{n}=$ 175) have a higher median but lower mean retweets (median $=8$, mean $=34$, standard deviation $=83$, $\mathrm{IQR}=3-24)$ than those which do not (median $=7$, mean $=48$, standard deviation $=348, \mathrm{IQR}=2-23)$. Considering number of hashtags shows that tweets with more hashtags have more retweets (Table 5).

Table 5: Number of hashtags

\begin{tabular}{l|r|r|r|l|r}
\hline Number of hashtags & Mean & Std dev & Median & IQR & Number of tweets \\
\hline none & 48 & 348 & 7 & $2-23$ & 833 \\
\hline 1 & 23 & 49 & 7 & $3-21.5$ & 128 \\
\hline 2 & 50 & 127 & 14 & $3-24$ & 29 \\
\hline 3 or more & 82 & 148 & 17 & $4-62.25$ & 18 \\
\hline
\end{tabular}




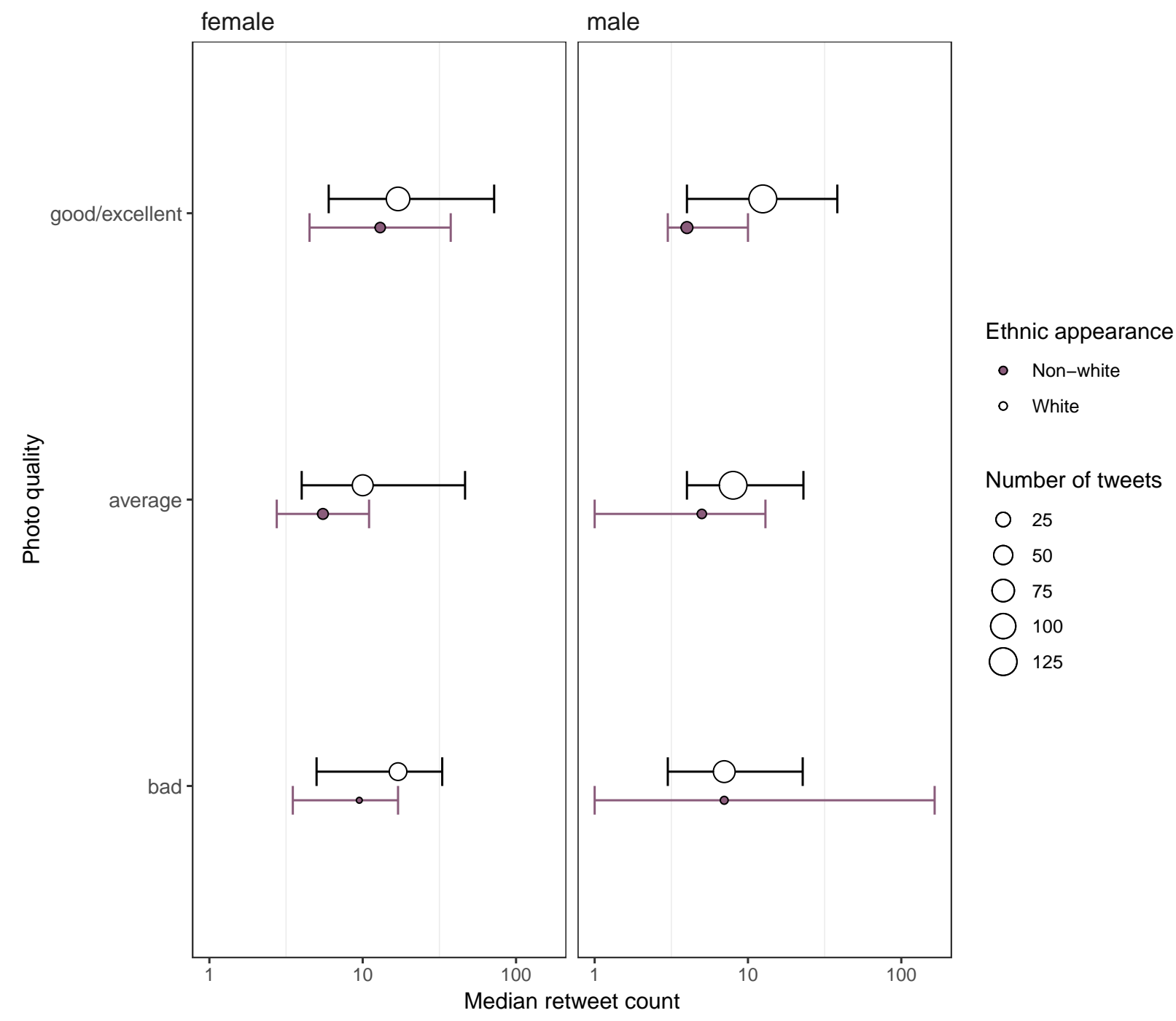

Figure 2: Retweets for different image quality by gender and ethnic appearance 


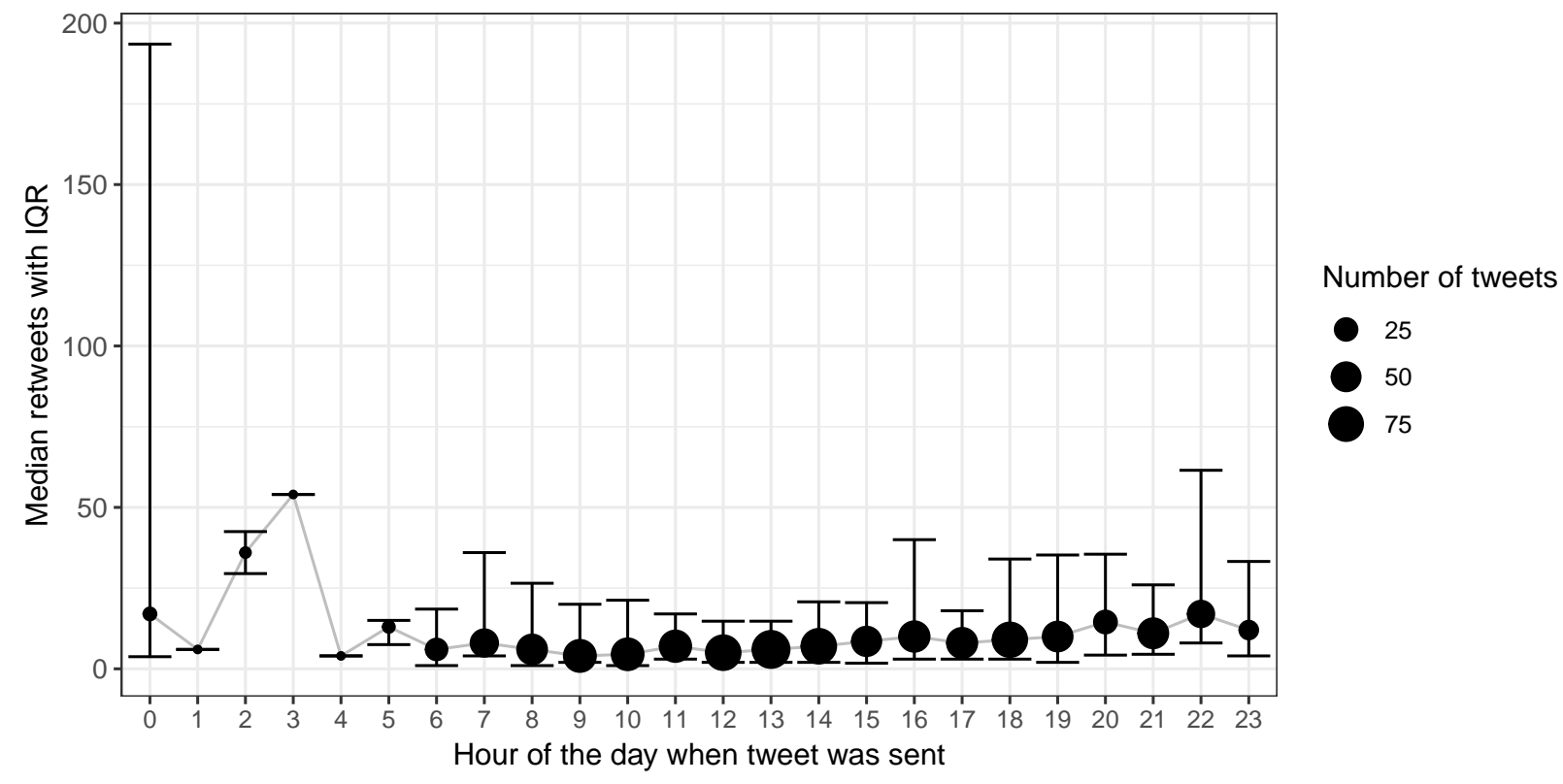

Figure 3: Retweets by hour of day

Besides the number of hashtags, it's content is also important. Thematic analysis was used to group hashtags into 3 overarching themes, and a miscellaneous "other" category (Table 6).

Table 6: Types of hashtags used (*some tweets have more than one type of hashtag)

\begin{tabular}{l|r|l}
\hline Hashtag type & No. of tweets & Examples \\
\hline location & 99 & \#Chorlton or \#Bury \\
\hline \#missing & 73 & \#Missing or \#MissingPerson \\
\hline police force or number & 10 & \#101 or \#GMP \\
\hline other & 15 & \#RT or \#update or \#helpfindjohn or \#thankyou \\
\hline
\end{tabular}

Figure 5 illustrates the different median retweets for different types of hashtags. Hashtags that mention location have a higher median retweet than tweets that do not have a location hashtag. On the other hand, using a hashtag for a police number (\#101 or \#999) and tweets using \#missing have lower median retweets than tweets which do not have these hashtags. "Other" is made up of the following hashtags: 4 referred to names (eg: \#FindCorrie or \#TessBlandamer), 2 expressed thanks (eg \#thankyou), 8 were abbreviations (eg: \#mufc, \#INPT1, \#whp) and two updates (\#Update and \#LatestNews).

Thematic coding to extract commonly used phrases which may act as templates identified initially 130 templates, which were further grouped into 12 overarching categories. About a quarter of tweets $(25.4 \%, \mathrm{n}$ $=255$ ) were completely original (no resemblance to any template), while the rest were coded into these 12 categories described in Table 7.

Figure 6 illustrates the different median retweets for different templates. Tweets that have the "have you seen...", "urgent appeal:", "police/etc are appealing for..." have much higher median retweets than tweets that do not have such phrasing. Tweets that mention that a missing person is high risk, and tweets that ask explicitly to "please retweet" also have higher median retweets than those which do not. Tweets that do not use any of these templates (original phrasing), tweets that use "can you help?" and tweets that use asterisks for emphasis (**missing**) have lower median retweets than tweets that do not use these templates.

From the AFINN sentiment lexicon we calculated scores for each tweet. Overall, the mean score was -1.56, and the median -2 . The lowest score was -10 and the highest was 4 . Scores follow a left-skewed distribution 


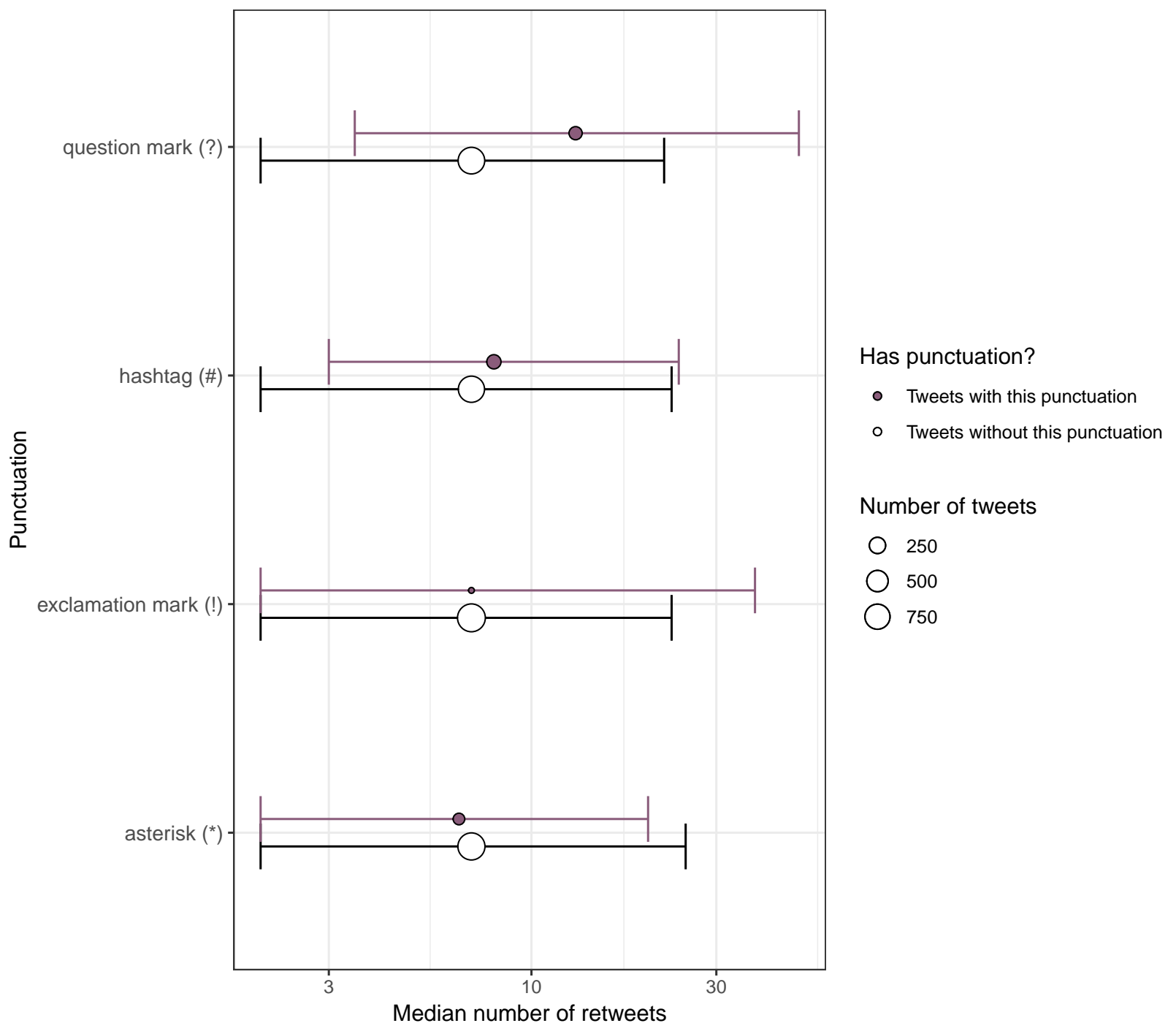

Figure 4: Retweets for the use of different punctuation 


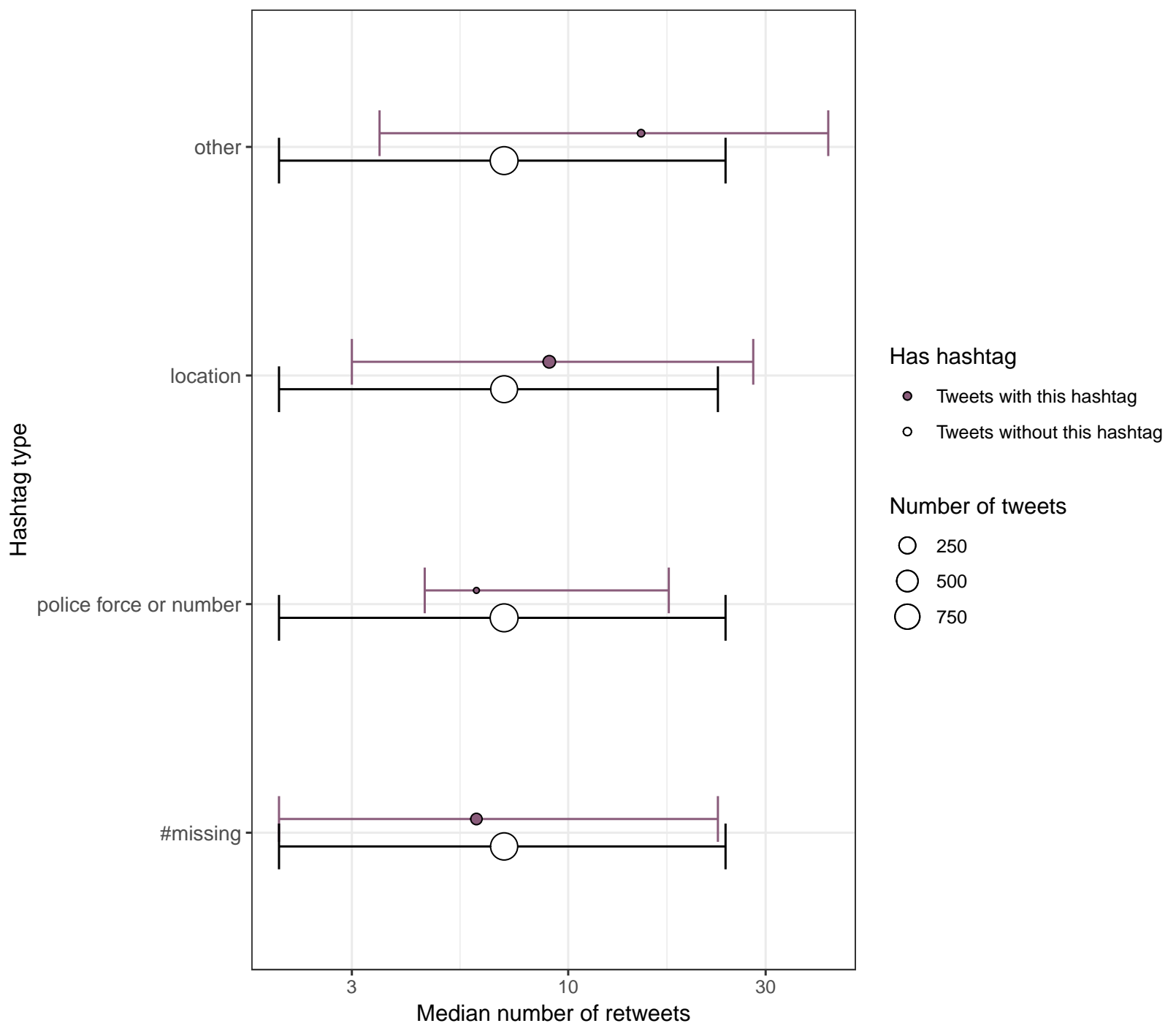

Figure 5: Retweets for different hashtag types 


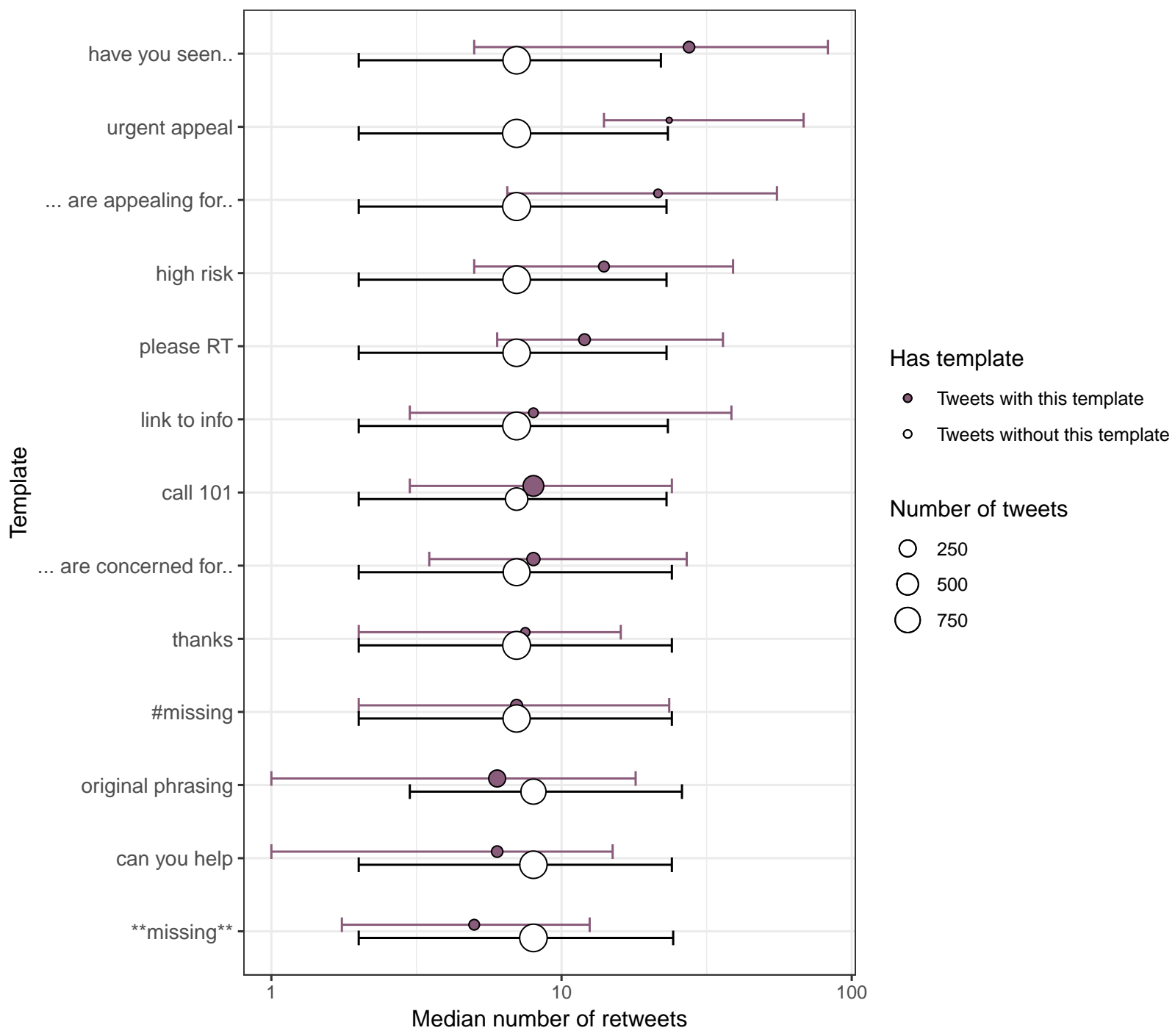

Figure 6: Retweets for different templates 
Table 7: Examples of templates

\begin{tabular}{|c|c|c|}
\hline Template & No. of tweets & Example \\
\hline call 101 & 449 & $\begin{array}{l}\text { "Police have released a new photograph of a high risk missing } \\
\text { teenager. [OTHER 661] you see [HIS/HER] please contact the } \\
\text { police. https://t.co/knlDpYw50K" }\end{array}$ \\
\hline original phrasing & 255 & $\begin{array}{l}\text { "\*\*`*[OTHER_204] - [OTHER_880] [OTHER_913] } \backslash^{*} \backslash^{*} \backslash^{*} \\
\text { https://t.co/oSOQmZlC03" }\end{array}$ \\
\hline ... are concerned for.. & 115 & $\begin{array}{l}\text { "Officers are growing increasingly concerned as to the } \\
\text { whereabouts of a 43-year-old man missing from Salford. } \\
\text { http://t.co/Zwg5aYzJxo" }\end{array}$ \\
\hline \#missing & 83 & $\begin{array}{l}\text { "\#Missing cont. \&gt;\&gt;\&gt;\&gt;Brian possibly in the location } \\
\text { of [OTHER_61] woods. If you see anybody matching the } \\
\text { description please call us on 101." }\end{array}$ \\
\hline please RT & 77 & $\begin{array}{l}\text { "We need your help to find missing [OTHER_369] } \\
\text { [OTHER_370] } 87 \text { yrs. [OTHER_429] seen in [OTHER_224] } \\
101 \text { if seen please [OTHER_51] @gmpchorlton } \\
\text { http://t.co/pJdb4oa5cX" }\end{array}$ \\
\hline can you help & 72 & "Missing - [OTHER_277] you help? http://t.co/4h816ZIrsi" \\
\hline have you seen.. & 72 & $\begin{array}{l}\text { "Thomas [OTHER_929] } 57 \text { years currently missing from } \\
\text { Crumpsall. [OTHER_422] you seen [HIM/HER] ring \#101 1/2" }\end{array}$ \\
\hline high risk & 58 & $\begin{array}{l}\text { "HIGH [OTHER_122] \#MISSING: [OTHER_502] you seen this } \\
\text { girl? [OTHER_503] Kenworthy. [OTHER_217] ring } 101 \text { if you } \\
\text { have any information. Thanks! http://t.co/DDslkiYCgq" }\end{array}$ \\
\hline$\backslash * \backslash * \operatorname{missing} \backslash^{*} \backslash *$ & 56 & $\begin{array}{l}\text { "\*MISSING } \backslash *[\text { OTHER_2101] last seen [OTHER_1519] } \\
\text { wearing a grey tracksuit, white t-shirt and trainers. } \\
\text { [OTHER_290] information please get in touch on '101'" }\end{array}$ \\
\hline link to info & 40 & 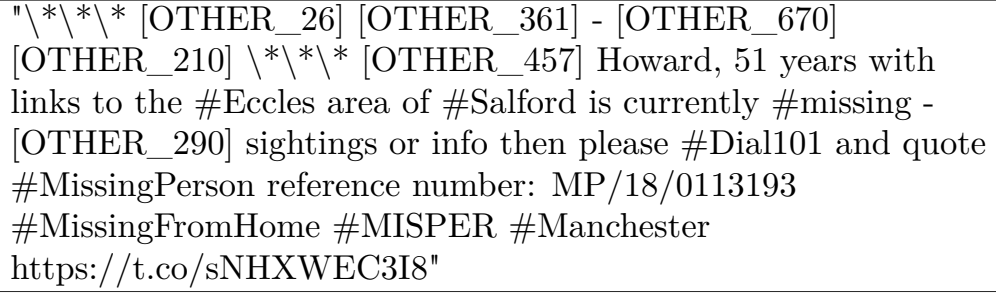 \\
\hline thanks & 34 & $\begin{array}{l}\text { "Mark [OTHER_1580] from Heywood, missing from home since } \\
\text { 29/06/15 believed to still be around [OTHER_1541] area if seen } \\
\text { please call } 101 \text { \#Thank you" }\end{array}$ \\
\hline ... are appealing for.. & 26 & $\begin{array}{l}\text { "Police are appealing for the public's help to find a man missing } \\
\text { from Rochdale. https://t.co/y5XQGhNGN6" }\end{array}$ \\
\hline urgent appeal & 12 & $\begin{array}{l}\text { "Urgent, please RT. [OTHER_204] Female, \#Atherton } \\
\text { [NUMBER_447], shoulder [OTHER_1485] [OTHER_42] Hair, } \\
\text { [OTHER_1488] T-Shirt, [OTHER_10] Trousers. [OTHER_121] } \\
\text { [OTHER_803] please ring 101" }\end{array}$ \\
\hline
\end{tabular}

with a left tail. There does not seem to be much of a relationship between sentiment score and retweet count (Pearson's product-moment correlation $=0.01, \mathrm{p}$-value $=0.65)$.

Figure 7 shows results from manually coded sentiment categories. Tweets coded scared or worried have higher median retweets than those not coded with these sentiments, followed by hopeful tweets. Hopeless and negative sentiment tweets have the much lower median retweets than tweets not coded with these sentiments. Mean Afinn sentiment score does not seem associated with manually coded sentiment. While 'willing to engage' tweets have a higher mean score (more positive), so do the tweets coded as 'negative' by the manual coder. The tweets coded 'hopeful' in the manual coding process have the lowest mean score $(-2.05)$, which is difficult to reconcile. 


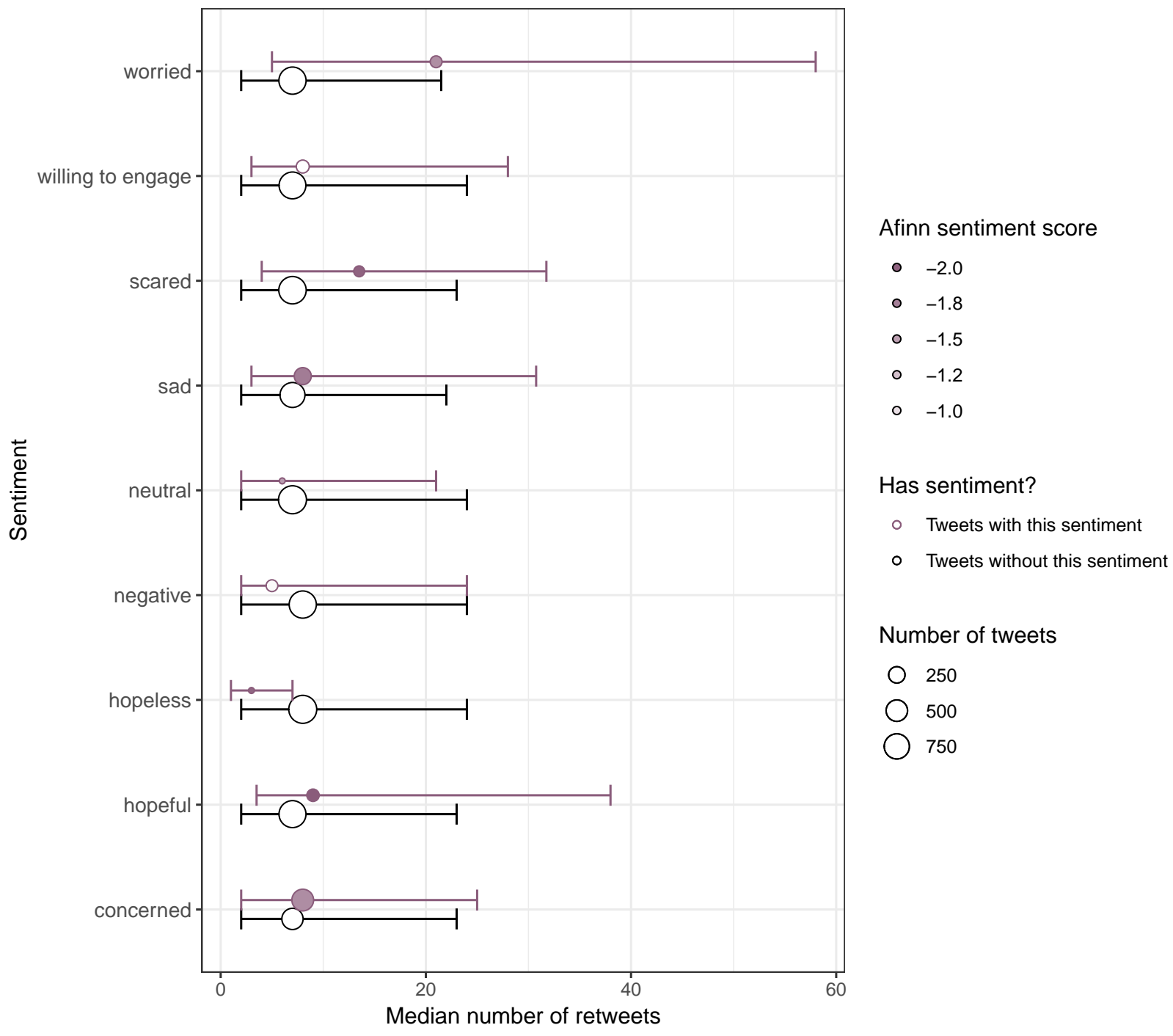

Figure 7: Retweets for different sentiments 


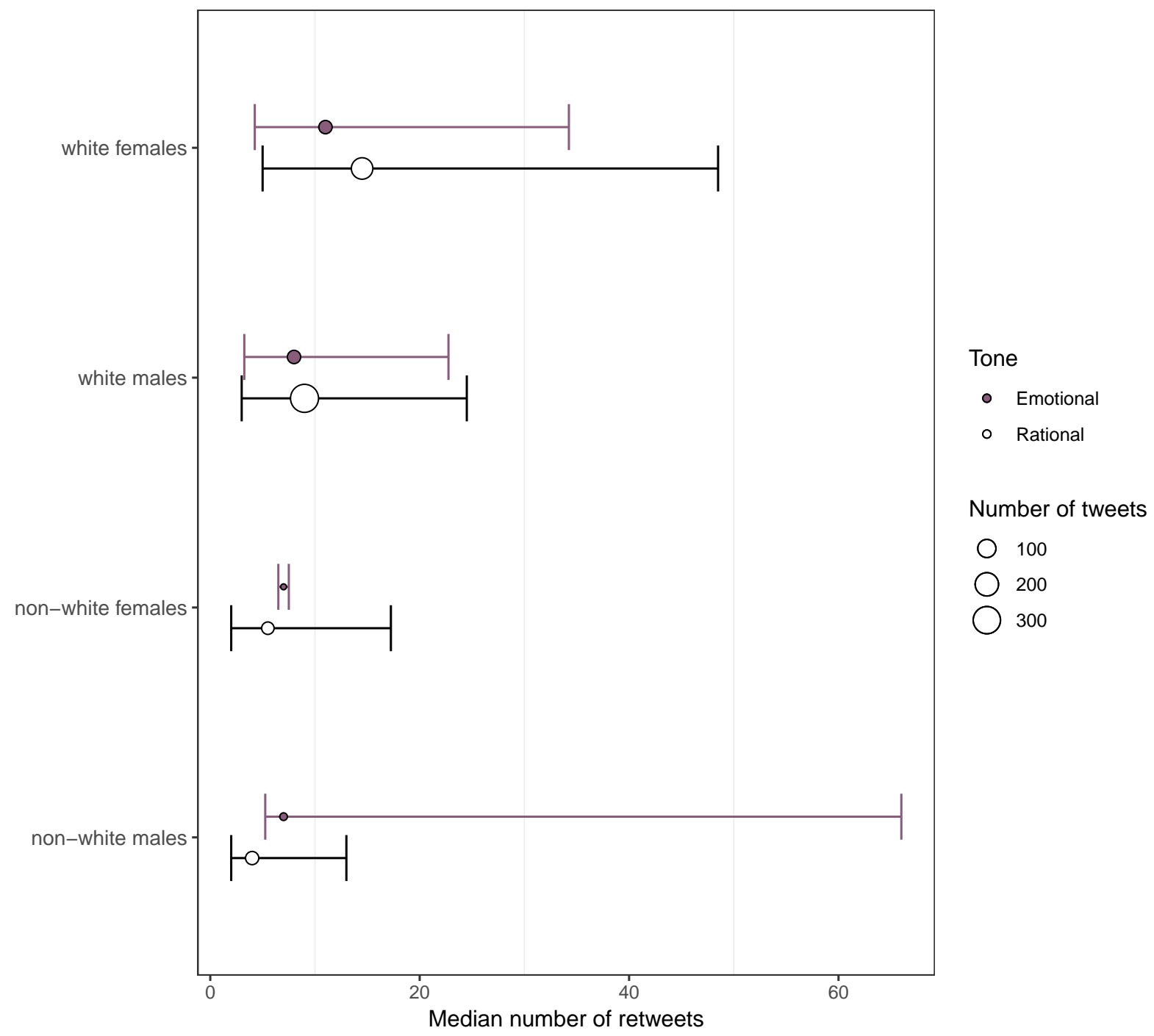

Figure 8: Retweets for different tones

Regarding tone, 871 tweets were coded as rational, and 137 as emotional. Altogether, tweets with an emotional tone have more retweets (median $=9$, mean $=141.85, \mathrm{sd}=831.04$, IQR $=3-30)$, than those with a rational tone (median $=7$, mean $=30.59, \mathrm{sd}=86.33, \mathrm{IQR}=2-23)$ (median $=7$, mean $=30.6$, sd $=86.3, \mathrm{IQR}=21)$. However, when separating out differences by gender and ethnic appearance, we see that for white missing persons rational tone have higher retweets, while for non-white missing persons emotional have higher number of retweets (Figure 8).

\section{Features of the account}

Finally we consider account characteristics. Number of daily tweets and age of account do not seem associated with retweets of appeals. Low activity accounts such as @GMPSaddleworth can have high RTs, and high tweet activity accounts can have low RTs, such as @GMPRadcliffe. While the oldest account (@gmpolice) has a high mean number of RTs, so does the youngest account (@gmpcheadle). Number of followers has a slight association, mostly driven by the two most followed accounts, which are @gmpolice and @GMPCityCentre 


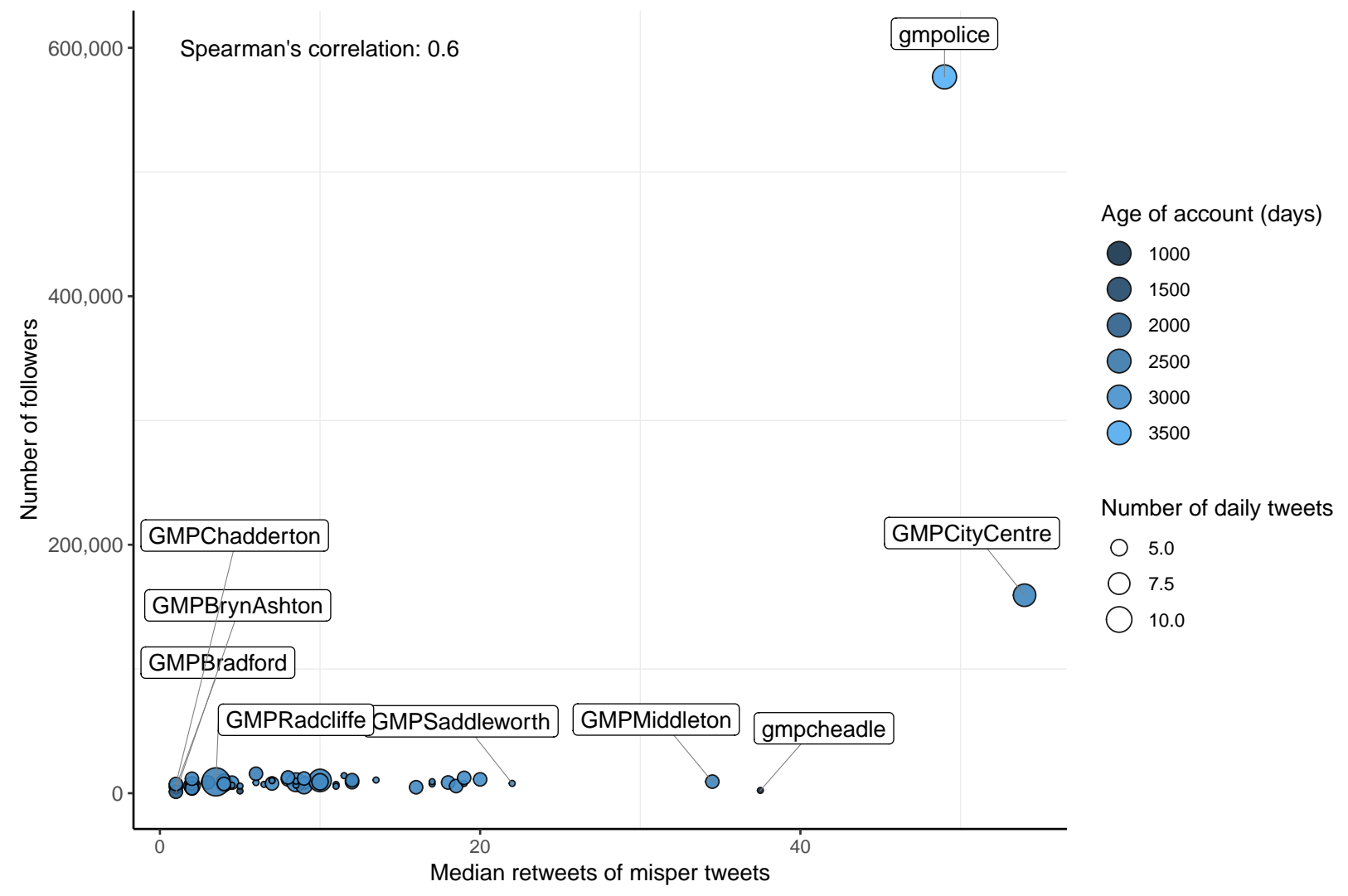

Figure 9: Account chatracteristics

(Figure 9).

\section{Accounting for exposure time}

Finally, we include an exploratory multivariable associational model, in order to be able to account for some tweets having been online longer than others. Exposure time is measured in days elapsed since the tweet was created, and we use this variable as an offset (a constant term in the linear predictor which is not estimated), useful for measuring rate data (retweets per exposures (days)).

The Poisson model shows evidence of overdispersion (Pearson Ch2 $=143778.85$, Dispersion $=247.47$ ), further indicated by the observed variance (101360.73) greatly exceeding the expected variance (58.45). Therefore we consider also a Poisson model with scaled standard error. The estimates for all variables from both models are illustrated in Figure 10.

While it is common to interpret the incident rate ratios as estimates of effect size between the dependent variables (in this case tweet features) and independent variable (retweets), doing so can lead to mistaken interpretations of these estimates in line with the 'Table 2 fallacy' (Westreich and Greenland 2013). As we are not modeling causal relationships, we will not interpret the incidence rate ratios. Instead we will look only for sign change between the findings of our bivariate analyses and what we see from the two models above. Accounting for exposure time and including all features in a single model simultaneously does not seem to change our conclusions from the earlier descriptive findings for the following features: men still have fewer retweets, while white missing persons have more. Regular photos and multiple photos have more retweets. Rational tweets have fewer retweets than emotional. Templates that follow "have you seen...?", contain a link, or ask to be retweeted have more retweets than tweets without these templates, while tweets 


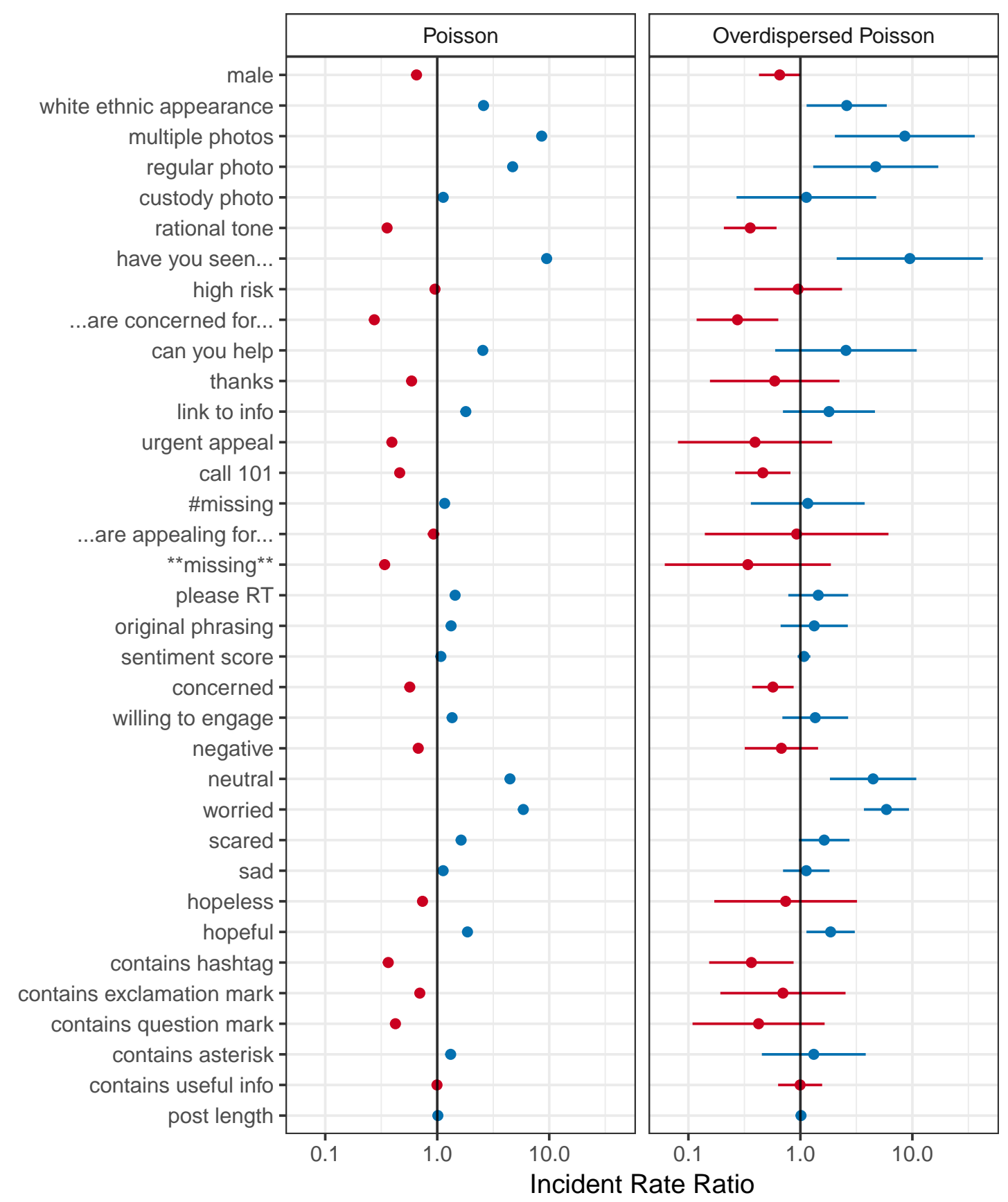

Figure 10: Multivariable models with offset 
using **missing** template have fewer retweets than those without this. However the estimates for the use of different punctuations (use of asterisk or question mark) change signs between the models and the bivariate analysis. Finally, worried, scared, willing to intervene, and hopeful tweets all remain positive (more retweets than those without these sentiments) while negative and hopeless tweets remain negative (fewer retweets than those without these sentiments).

\section{Discussion}

To explore how police tweet missing persons appeals and how the public engage with these, we analysed 1008 tweets made by GMP, using retweets as a measure of engagement and spread. Our results constitute a first empirical insight into police appeals for information about missing persons made on Twitter, and public engagement with these appeals. We pick up below our key findings to discuss how current tweets are structured, and what factors should be considered when investigating what features might affect public engagement.

Firstly we found that the majority of tweets receive very low engagement, with $18.35 \%$ ( $\mathrm{n}=185$ ) of the tweets getting zero or one retweets. This suggests that there are some current strategies which receive very limited impact. On the other hand, there are quite a few tweets which receive much retweeting activity. $12.1 \%(\mathrm{n}=122)$ of tweets were considered as outliers (outside 1.5 times the interquartile range above the upper quartile), 78 of these are extreme outliers (outside 3 times the interquartile range above the upper quartile). Further work should explore in depth why so many tweets receive little to no engagement and what makes others "go viral".

Our key focus is on features of tweets which can be acted upon by police and other organisations making these appeals. Below we draw some preliminary conclusions about what could be considered when writing Twitter appeals. One decision police make is to include a photo with the appeal, and what sort of photo to choose. We find in our sample that tweets with a regular photo or multiple photos had higher engagement than tweets with a custody photo, or tweets without any photo. This is in line with previous literature, which found an association between photo valence and retweeting (Stefanone, Egnoto, and Fu 2015). Another explanation is that a custody photo does not resonate with people on a personal level, something identified by previous work as important motivation for sharing (Juncu and Glorney 2019). This is interesting but we must keep in mind that the appeal has many aims, one of which is to reach far and wide, while another is to contain valuable information to help identify the missing person. Therefore, a custody image might still be better than no image, as it will facilitate recognition of the missing person amongst those who do see the appeal. Regarding photo quality, we did not find more retweets with improved quality, but did find that the different in retweets between white and non-white men increases with each improvement in the quality of the photograph.

Nothing in our sample suggested that timing of tweet made a difference to retweeting. As we know that timeliness of information is crucial in missing persons investigations (Smith and Shalev Greene 2015) we suggest that appeals are made as early as possible, rather than trying to optimise the best time to tweet.

Considering which templates to use, tweets following the "have you seen..." template, tweets including a link, and tweets that ask followers to "please retweet" received more engagement. These could be interpreted as calls-to-action templates, which Lopez (2014) associated with increased engagement. Our findings on sentiment may be harder to implement, and we recognise that being mindful of the sentiment that a user might feel is a very difficult task, on top of trying to fit as much information as possible into a 280 character appeal, making this difficult to action. However, some results here might be easier to distinguish, for example how hopeful sentiment tweets in our sample got more and hopeless ones got fewer retweets. An example of a tweet coded hopeful illustrates what such tweets might sound like:

"FIRSTNAME LASTNAME. Missing from \#LOCATION for 3 years. Today is his birthday. Have YOU seen this man? Please don't stop the RTs. LINK"

On the other hand, an example of a tweet coded hopeless is: 
"FIRSTNAME LASTNAME has been missing for a year now LINK"

It is possible that injecting 'hope' into these tweets can lead to more sharing.

The difference in engagement with rational versus emotional tweets has been highlighted by previous literature as something which affects retweets but with no clear consensus on how (Lopez 2014; Xu and Zhang 2018). In fact, we also find duality in our results. Overall it seems that in our sample emotional tweets have higher retweets than our rational tweets. However, when we separate out gender and ethnicity, we can notice that for tweets about white men and women, rational tone tweets have more retweets, but for non-white men and women, it is emotional tweets which do.

While the above presents an exciting empirical insight into police appeals for missing persons on social media, our study also has some limitations to keep in mind. First, we already mentioned about the data issues, in particular that selection into our sample requires that the tweet was not deleted, and this is likely to be related to features and outcome under study. This means that we have a biased sample. Not only that, but do not understand how this bias takes effect, since although posts about people who have returned to home are supposed to be taken down, we can see from our data that this is not always the case. Our results therefore are purely descriptive, and are meant as an indication into what sorts of characteristics of the features we identified through our literature review we even see in police tweets about missing persons. We recommend that future work considers a prospective study design, or employs a randomised control trial approach to be able to speak to the causal relationships between the features identified here, and public engagement. Clearly, there is an imperative to identify effective strategies so that we can help find missing persons as quickly and efficiently as possible. By employing a randomised control design we can collect robust evidence to identify causal mechanisms behind what works in promoting wide sharing and engagement with Twitter appeals. Longitudinal designs could allow insight into different temporal patterns in retweeting, rather than a final total retweets measure as we have here. This would allow us to employ causal inference methods to consider the time-varying features of the accounts as well, and answer questions such as: "as accounts gain more followers, do their tweets become more retweeted?". Another limitation, which would not be addressed by the above study designs is that all these results are based on data from Twitter, and there are other social media platforms out there, to which these findings should ideally generalise. However, Juncu and Glorney (2019) found there not to be too much of a difference between those who use Facebook and Twitter to share information about missing people, so this may be a less serious issue. Finally, we have looked at engagement as an outcome measure, but future work could explore the effectiveness of these appeals in leading to valuable information or helping to locate the missing person, as well as the ethical implications and risks introduced by public sharing of missing people's personally identifiable information on social media platforms. While wide sharing might be a desired outcome to help find the missing person, it can compound the problem of the limited scope the investigators have to withdraw the information they had released, once the person is found (Holmes 2016).

\section{Conclusion}

In this paper we explored appeals for information about missing persons made on Twitter by greater Manchester police. We did so in order to uncover how the police currently construct such appeals, and whether we can infer any structure in the practice. We find that there is some structure, but there is also variation in how these messages are crafted, as well as in other features such as the type and quality of photo used, the phrasing and punctuation used, and the perceived sentiment that results. We considered how engagement, measured as retweets varies between these differently structured tweets, and draw conclusions about what we think might be important to follow up. In particular we present exploratory results, which serve the basis for further confirmatory work. Therefore, the contribution of this paper is two-fold. First, we provide an insight into how appeals for information for missing people are shared by a major UK police force. We can see that although many of these messages follow a template, many others do not, and the choice of which template to follow, as well as other decisions around what information, images, or other features to include might have consequences for how the message is viewed and shared by audiences. By presenting the first look into these 
practices, our exploratory research serves as a foundation for future confirmatory work to build upon. In particular we have laid out a set of features which are used in missing persons appeals on social media that can inform studies with a prospective design, or experimental randomised control trial approach to further uncover causal relationships, and make recommendations for good practice in appeals for missing persons on Twitter from police, and other organisational accounts. Secondly, and relatedly, we serve as a reference point for an issue that is internationally relevant, affecting police and other organisations worldwide. Other countries with growing demand on their services from increasing numbers of people reported missing paired with restrictions on resources must also consider how to optimise their messages on social media so it reaches far and wide. This exploration is applicable and replicable in a way to inform more work internationally, with the ultimate aim to assist organisations and governments in safeguarding their vulnerable citizens.

\section{Acknowledgements}

This work was funded by the Manchester Statistical Society Campion Grant. All code was written in R (version 3.5.1) (R Core Team 2018), using the following packages: Wickham et al. (2019), Wickham et al. (2018), Slowikowski (2018), Xie (2018), Spinu, Grolemund, and Wickham (2018), Allaire et al. (2018), Wickham (2018), and Wickham and Henry (2018). Code for this paper can be found on www.github.com/maczokni/misperTweetsCode. The authors would like to thank Aiden Sidebottom, Freya O'Brien, Joe Apps, Jane Hunter, Emily Moir, and Juanjo Medina for valuable comments on earlier drafts of this manuscript.

\section{References}

ACPO. 2013. "Guidelines for the Safe Use of the Internet and Social Media by Police Officers and Police Staff." http://library.college.police.uk/docs/ACPO/safe- use-of-the-Internet-Feb-2013.pdf.

Allaire, JJ, Yihui Xie, Jonathan McPherson, Javier Luraschi, Kevin Ushey, Aron Atkins, Hadley Wickham, Joe Cheng, Winston Chang, and Richard Iannone. 2018. Rmarkdown: Dynamic Documents for R. https: //CRAN.R-project.org/package=rmarkdown.

Artwick, C. G. 2010. "White, Black and Missing on Twitter: Social Media Highlight Disparities in News Coverage of Two Missing Virginia Women." Quill, 18-22.

Biehal, Nina, Fiona Mitchell, and Jim Wade. 2003. "Lost from View: Missing Persons in the Uk." The Policy Press 17.

Cha, M., H. Haddadi, F. Benevenuto, and K. P. Gummadi. 2010. Measuring User Influence in Twitter: The Million Follower Fallacy. In Proceedings of the Fourth International AAAI Conference on Weblogs; Social Media.

Chen, R, and Y Sakamoto. 2013. "Perspective Matters: Sharing of Crisis Information in Social Media." In 46th Hawaii International Conference on System Sciences, 2033-41.

Chiefs of Police, International Association of. 2018. "Missing Persons: Volunteers Supporting Law Enforcement." https://www.theiacp.org/sites/default/files/2018-09/missing-persons.pdf.

Crawford, Adam. 2013. "Chapter 11: The Police, Policing and the Future of the 'Extended Policing Family'." In The Future of Policing, edited by Jennifer M Brown, 173-90. New York: Routledge.

Crump, J. 2011. "What Are the Police Doing on Twitter?" Social Media, the Police and the Public, Policy E Internet 3 (4): 7 .

Cvetojevic, S., and H. H. Hochmair. 2018. "Analyzing the Spread of Tweets in Response to Paris Attacks." Computers, Environment and Urban Systems 71: 14-26. 
Dai, M., W. He, X. Tian, A. Giraldi, and F. Gu. 2017. "Working with Communities on Social Media: Varieties in the Use of Facebook and Twitter by Local Police." Online Information Review 41 (6): 782-96.

Denef, S., P. S. Bayerl, and N. Kaptein. 2013. "Social Media and the Police- Tweeting Practices of British Police Forces During the August 2011 Riots." In CHI. Paris, France.

Earl, Jennifer, McKee HurwitzHeather, Mejia MesinasAnalicia, Margaret Tolan, and Ashley Arlotti. 2013. "This Protest Will Be Tweeted: Twitter and Protest Policing During the Pittsburgh G20." Information, Communication 8 Society 16 (4): 459-78.

Ellingwood, H. 2016. "A Better Estimation of Police Costs by Offence Types." Wollaston Lake, SK: Public Safety Canada/Sécurité Publique Canada. https:/www.publicsafety.gc.ca/cnt/rsrcs/pblctns/2015-r018/ index-en.aspx.

Fernandez, M., A. E. Cano, and H. Alani. 2014. "Policing Engagement via Social Media." In: City Labs Workshop - SociInfo, November, 10-13.

Ferrara, E., and Z. Yang. 2015. "Quantifying the Effect of Sentiment on Information Diffusion in Social Media." PeerJ Computer Science 1 (26): 1-15.

Fyfe, Nicholas R., Olivia Stevenson, and Penny Woolnough. 2015. "Missing Persons: The Processes and Challenges of Police Investigation." Policing and Society 25 (4): 409-25. https://doi.org/10.1080/10439463. 2014.881812.

Garside, R., A. Silvestri, and H. Mills. 2013. "UK Justice Policy Review." https://www.crimeandjustice. org.uk/sites/crimeandjustice.org.uk/files/UK.

Gilchrist, K. 2010. “'Newsworthy' Victims?” Feminist Media Studies 10 (4): 373-90.

Graneheim, U. H., and B. Lundman. 2004. "Qualitative Content Analysis in Nursing Research: Concepts, Procedures and Measures to Achieve Trustworthiness." Nurse Education Today 24 (2): 105-12. https: //doi.org/10.1016/j.nedt.2003.10.001.

Guest, G., K. M. MacQueen, and E. E. Namey. 2011. Applied Thematic Analysis. London: Sage Publications.

Hilbe, J. 2014. Modeling Count Data. Cambridge: Cambridge University Press.

Holmes, L. 2016. "Missing Person Appeals: A Uk Perspective." In Handbook of Missing Persons, 19-35. Cambridge: Springer.

Hong, L., O. Dan, and D. B. Davison. 2011. "Predicting Popular Messages on Twitter." In $W W W$. Hyderabad, India.

Jeanis, M. N., and R. A. Powers. 2017. "Newsworthiness of Missing Persons Cases: An Analysis of Selection Bias, Disparities in Coverage, and the Narrative Framework of News Reports." Deviant Behavior 38 (6): 668-83.

Jenders, Maximilian, Gjergji Kasneci, and Felix Naumann. 2013. "Analyzing and Predicting Viral Tweets." In Proceedings of the 22nd International Conference on World Wide Web, 657-64. ACM.

Juncu, S., and E. Glorney. 2019. Exploring the Factors That Impact Individuals' Willingness to Share Missing People Appeals on Facebook and/or Twitter. Manuscript in preparation.

Kim, E., J. Hou, J. Y. Han, and I. Himelboin. 2016. "Predicting Retweeting Behavior on Breast Cancer Social Networks: Network and Content Characteristics." Journal of Health Communication 21 (4): 479-86.

Kwon, K Hazel, C Chris Bang, Michael Egnoto, and Raghav RaoH. 2016. "Social Media Rumors as Improvised Public Opinion: Semantic Network Analyses of Twitter Discourses During Korean Saber Rattling 2013." Asian Journal of Communication 26 (3): 201-22.

Lampinen, James Michael, Christopher S Peters, and Vicki S Gier. 2012. "Power in Numbers: The Effect of Target Set Size on Prospective Person Memory in an Analog Missing Child Scenario." Applied Cognitive Psychology 26 (5): 702-8. 
Lopez, L. L. 2014. "Message Appeals Used by Nonprofits on Twitter to Increase Public Engagement." PhD thesis, LSU Master's Theses. 2386.

Lovell, J. S. 2001. "Media Power and Information Control: A Study of Police Organizations \& Media Relations." PhD thesis, Rutgers University, School of Criminal Justice.

Meijer, A., and M. Thaens. 2013. "Social Media Strategies: Understanding the Differences Between North American Police Departments." Government Information Quarterly 30 (4): 343-50.

Min, Seong-Jae, and John C Feaster. 2010. "Missing Children in National News Coverage: Racial and Gender Representations of Missing Children Cases." Communication Research Reports 27 (3): 207-16.

National Crime Agency. 2017. "UK Missing Persons Bureau: Missing Persons Data Report 2015/2016". http://nationalcrimeagency.gov.uk/publications/876-missing-persons-data-report-2015-2016-1/file.

—. 2019. Missing Persons Data Report 2016/201\%. https://www.nationalcrimeagency.gov.uk/ who-we-are/publications/304-2016-17-ukmpu-data-report-v1/file.

Naveed, N., T. Gottron, J. Kunegis, and Che AlhadiA. 2011. "Bad News Travel Fast: A Content-Based Analysis of Interestingness on Twitter." In Proceedings of the 3rdInternational Web Science Conference, 15-17. 2011, Koblenz, Germany: June.

Petonito, G., G. W. Muschert, D. C. Carr, J. M. Kinney, E. J. Robbins, and J. S. Brown. 2012. "Programs to Locate Missing and Critically Wandering Elders: A Critical Review and a Call for Multiphasic Evaluation." The Gerontologist 53 (1): 17-25.

Police, Greater Manchester. 2019. "“Twitter'” http://www.gmp.police.uk/content/WebsitePages/ 6C386353FE565CCF80257B48004ABAF5.

Policing, College of. 2019. "Authorised Professional Practice Content: Major Investigation and Public Protection: Missing Persons." https://www.app.college.police.uk/app-content/major-investigation-and-public-protection/ missing-persons/.

R Core Team. 2018. R: A Language and Environment for Statistical Computing. Vienna, Austria: R Foundation for Statistical Computing. https://www.R-project.org/.

Rees, Gwyther. 2011. "Still Running 3: Early Findings from Our Third National Survey of Young Runaways'. The Children's Society." https://www.childrenssociety.org.uk/sites/default/files/tcs/still_running_3_full_ report_final.pdf.

Rijt, A. van de, H. G. Song, E. Shor, and R. Burroway. 2018. "Racial and Gender Differences in Missing Children's Recovery Chances." PLoS One 13: 12.

Rowe, M., S. Angeletou, and H. Alani. 2011. "Predicting Discussions on the Social Semantic Web." In 8th Extended Semantic Web Conference (Eswc 2011). Heraklion, Greece.

Rowe, M., S. Angeletous, and H. Alani. 2011. "Anticipating Discussion Activity on Community Forums." In IEEE International Conference on Privacy, Security, Risk, and Trust, and Ieee International Conference on Social Computing, 315-22.

Shalev Greene, Karen, and Francis Pakes. 2013. "The Cost of Missing Person Investigations: Implications for Current Debates." Policing: A Journal of Policy and Practice 8 (1): 27-34.

Sharp-Jeffs, N. 2016. "Hidden Links? Going Missing as an Indicator of Child Sexual Exploitation." In Missing Persons, 38-47. Routledge.

Slowikowski, Kamil. 2018. Ggrepel: Automatically Position Non-Overlapping Text Labels with 'Ggplot2'. https://CRAN.R-project.org/package=ggrepel.

Smedley, R. M., and N. S. Coulson. 2018. "A Practical Guide to Analysing Online Support Forums." Qualitative Research in Psychology, 1-28.

Smith, R., and Shalev GreeneK. 2015. "Recognizing Risk: The Attitudes of Police Supervisors to the Risk Assessment Process in Missing Person Investigations." Policing: A Journal of Policy and Practice 9 (4): $352-61$. 
Spinu, Vitalie, Garrett Grolemund, and Hadley Wickham. 2018. Lubridate: Make Dealing with Dates a Little Easier. https://CRAN.R-project.org/package=lubridate.

Stefanone, M. A., M. J. Egnoto, and Y. Fu. 2015. "Image Attributes and Diffusion via Twitter : The Case of \#Guncontrol." In IEEE Computer Society 48th Hawaii International Conference on System Sciences, $1788-97$.

Stevenson, Olivia, Hestor Parr, Penny Woolnough, and Nick Fyfe. 2013. "Geographies of Missing People: Processes, Experiences, Responses [Online]."

Suh, B., L. Hong, P. Pirolli, and E. H. Chi. 2010. "Want to Be Retweeted? Large Scale Analytics on Factors Impacting Retweet in Twitter Network." In Second International Conference on Social Computing, 177-84. IEEE Computer Society.

Tapia, A. H., K. Bajpai, B. J. Jansen, J. Yen, and L. Giles. 2011. "Seeking the Trustworthy Tweet: Can Microblogged Data Fit the Information Needs of Disaster Response and Humanitarian Relief Organizations." In 8th International Iscram Conference, 1-10. Lisbon, Portugal: ISCRAM.

Tsoi, K. K. F., N. B. Chan, F. C. H. Chan, L. Zhnag, A. C. H. Lee, and Meng H.M. L. 2018. "How Can We Better Use Twitter to Find a Person Who Got Lost Due to Dementia?" Npj Digital Medicine, 1-14.

Van de Velde, B., A. Meijer, and V. Homburg. 2015. "Police Message Diffusion on Twitter: Analysing the Reach of Social Media Communications." Behaviour 83 Information Technology 34 (1): 4-16.

Westreich, Daniel, and Sander Greenland. 2013. "The Table 2 Fallacy: Presenting and Interpreting Confounder and Modifier Coefficients." American Journal of Epidemiology 177 (4): 292-98.

Wickham, Hadley. 2018. Scales: Scale Functions for Visualization. https://CRAN.R-project.org/package= scales.

Wickham, Hadley, Winston Chang, Lionel Henry, Thomas Lin Pedersen, Kohske Takahashi, Claus Wilke, and Kara Woo. 2018. Ggplot2: Create Elegant Data Visualisations Using the Grammar of Graphics. https: //CRAN.R-project.org/package=ggplot2.

Wickham, Hadley, Romain François, Lionel Henry, and Kirill Müller. 2019. Dplyr: A Grammar of Data Manipulation. https://CRAN.R-project.org/package=dplyr.

Wickham, Hadley, and Lionel Henry. 2018. Tidyr: Easily Tidy Data with 'Spread()' and 'Gather()' Functions. https://CRAN.R-project.org/package=tidyr.

Williams, G A, and F Finlay. 2019. "County Lines: How Gang Crime Is Affecting Our Young People." Archives of Disease in Childhood 104: 730-32.

Xie, Yihui. 2018. Knitr: A General-Purpose Package for Dynamic Report Generation in R. https://CRAN. R-project.org/package $=$ knitr.

Xu, W. W., and C. Zhang. 2018. "Sentiment, Richness, Authority, and Relevance Model of Information Sharing During Social Crises - the Case of \#Mh370 Tweets." Computers in Human Behaviours 89: 199-206.

Yan, J., J. Guszcza, M. Flynn, and C. S. P. Wu. 2009. "Applications of the Offset in Property-Casualty Predictive Modeling." In Casualty Actuarial Society E-Forum, 366.

Zarrella, Dan. 2009. The Social Media Marketing Book. " O’Reilly Media, Inc.". 